\title{
New cytotoxic steroids from the soft coral Clavularia viridis
}

\author{
Chang-Yih Duh ${ }^{a, b, *}$, I-Wen Lo $^{a}$, Shang-Kwei Wang ${ }^{c}$, Chang-Feng Dai ${ }^{d}$ \\ a Department of Marine Biotechnology and Resources, National Sun Yat-sen University, Kaohsiung, Taiwan \\ b Center of Asia-Pacific Marine Researches, National Sun Yat-sen University, Kaohsiung, Taiwan \\ c Department of Microbiology, Kaohsiung Medical University, Kaohsiung, Taiwan \\ d Institute of Oceanography, National Taiwan University, Taipei, Taiwan
}

\section{A R T I C L E I N F O}

Article history:

Received 6 January 2007

Received in revised form

16 March 2007

Accepted 16 March 2007

Published on line 30 March 2007

Keywords:

Steroids

Clavularia viridis

Cytotoxicity

\begin{abstract}
A B S T R A C T
Ten new cytotoxic steroids, stoloniferones $\mathrm{H}-\mathrm{Q}$ (1-10) were isolated from the methylene chloride solubles of the soft coral Clavularia viridis. The structures of the metabolites were elucidated on the basis of spectroscopic (IR, MS, and 1D and 2D NMR) analysis and their cytotoxicity against selected cancer cells was measured in vitro.
\end{abstract}

(c) 2007 Elsevier Inc. All rights reserved.

\section{Introduction}

The genus Clavularia has afforded many types of bioactive prostanoids, terpenoids and steroids [1-11]. As part of our search for bioactive substances from marine organisms, the soft coral Clauularia viridis Quoy and Gaimard (class Anthozoa, subclass Octocorallia, order Stolonifera) was studied because the $\mathrm{CH}_{2} \mathrm{Cl}_{2}$ extracts showed significant cytotoxicity to HT-29 (human colon adenocarcinoma), and P-388 (mouse lymphocytic leukemia) cell cultures as determined by standard procedures $[12,13]$. Bioassay-guided fractionations of the $\mathrm{CH}_{2} \mathrm{Cl}_{2}$ extracts resulted in the isolation of ten new cytotoxic steroids, stoloniferones H-Q (1-10).

\section{Experimental}

\subsection{General}

Optical rotations were determined on a JASCO DIP-181 polarimeter. UV spectra were obtained on a Shimadzu UV$160 \mathrm{~A}$ spectrophotometer, and IR spectra were recorded on a Hitachi 26-30 spectrophotometer. The NMR spectra were recorded on Bruker Avance $300 \mathrm{NMR}$ spectrometer at $300 \mathrm{MHz}$ for ${ }^{1} \mathrm{H}$ and $75 \mathrm{MHz}$ for ${ }^{13} \mathrm{C}$, respectively, in $\mathrm{CDCl}_{3}$ using TMS as internal standard. ESIMS spectra were obtained with a Bruker APX II mass spectrometer. Si gel 60 (Merck, 230-400 mesh)

\footnotetext{
* Corresponding author. Tel.: +886 7 5252000x5036; fax: +886 75255020.

E-mail address: yihduh@mail.nsysu.edu.tw (C.-Y. Duh). 
was used for column chromatography; precoated Si gel plates (Merck, Kieselgel $60 \mathrm{~F}_{254}, 0.25 \mathrm{~mm}$ ) were used for TLC analysis.

\subsection{Animal material}

The soft coral Clauularia viridis was collected at Green Island, off Taiwan, in July 2003, at a depth of 2-3 m and was stored for 1 month in a freezer until extraction. A voucher specimen, NSUGN-061, is deposited in the Department of Marine Biotechnology and Resources, National Sun Yat-sen University, Taiwan.

\subsection{Extraction and isolation}

The bodies of the soft coral $C$. viridis were freeze dried to give $1.90 \mathrm{~kg}$ of a solid, which was extracted with $\mathrm{CH}_{2} \mathrm{Cl}_{2}(5.0$ $1 \times 3)$. After removal of solvent in vacuo, the residue $(80 \mathrm{~g})$ was chromatographed over silica gel 60 using $n$-hexane and $n$-hexane/EtOAc mixtures of increasing polarity. Elution by $n$-hexane/EtOAc (60:40 v/v) afforded fractions containing compounds 8-10. Elution by $n$-hexane/EtOAc $(50: 50 \mathrm{v} / \mathrm{v})$ afforded fractions containing compounds 1-7. Compounds 1-7 were further purified by $\mathrm{C}_{18} \mathrm{HPLC}$ column using $\mathrm{MeOH} / \mathrm{H}_{2} \mathrm{O}$ (86:14 $\mathrm{v} / \mathrm{v}$ ) as solvent system. Compounds 8-10 were further purified by $\mathrm{C}_{18}$ HPLC column using $\mathrm{MeOH} / \mathrm{H}_{2} \mathrm{O}(88: 12 \mathrm{v} / \mathrm{v})$ as solvent system.
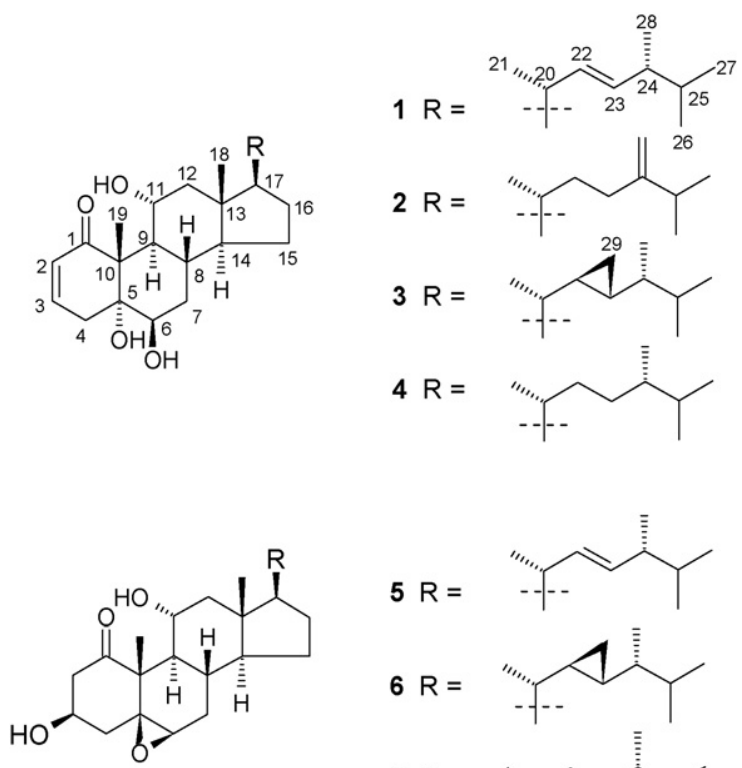

$5 \mathrm{R}=$

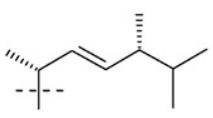

$6 \mathrm{R}=$<smiles>CC(C)[C@@H](C)C1C[C@H]1[C@@H](C)C(C)(C)C</smiles>

$7 \mathrm{R}=$<smiles>CC(C)[C@@H](C)CC[C@H](C)C(C)(C)C</smiles>

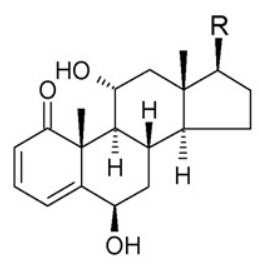<smiles>[R]O[Si]=[R]</smiles>

2.3.1. Stoloniferone $\mathrm{H}(1)$

Colorless amorphous solid; $[\alpha]_{\mathrm{D}}^{25}=+15.0\left(c=0.1, \mathrm{CHCl}_{3}\right)$; UV $(\mathrm{MeOH}) \lambda_{\max } 224 \mathrm{~nm}\left(\log \varepsilon\right.$ 3.56); IR $\nu_{\max } 3420,1653 \mathrm{~cm}^{-1} ;{ }^{1} \mathrm{H}$
NMR see Table $1 ;{ }^{13} \mathrm{C}$ NMR see Table 2; HRESIMS m/z 467.3134 (calcd for $\mathrm{C}_{28} \mathrm{H}_{44} \mathrm{O}_{4} \mathrm{Na}$, 467.3137).

\subsubsection{Stoloniferone I (2)}

Colorless amorphous solid; $[\alpha]_{D}^{25}=+12.0\left(c=0.1, \mathrm{CHCl}_{3}\right)$; UV (MeOH) $\lambda_{\max } 225 \mathrm{~nm}\left(\log \varepsilon\right.$ 3.58); IR $v_{\max } 3430,1656 \mathrm{~cm}^{-1} ;{ }^{1} \mathrm{H}$ NMR see Table $1 ;{ }^{13} \mathrm{C}$ NMR see Table 2; HRESIMS $\mathrm{m} / \mathrm{z} 467.3134$ (calcd for $\mathrm{C}_{28} \mathrm{H}_{44} \mathrm{O}_{4} \mathrm{Na}$, 467.3137).

\subsubsection{Stoloniferone J (3)}

Colorless amorphous solid; $[\alpha]_{D}^{25}=+26.0\left(c=0.1, \mathrm{CHCl}_{3}\right)$; UV $(\mathrm{MeOH}) \lambda_{\max } 224 \mathrm{~nm}(\log \varepsilon 3.46) ; \mathrm{IR} \nu_{\max } 3400,1658 \mathrm{~cm}^{-1} ;{ }^{1} \mathrm{H}$ NMR see Table $1 ;{ }^{13} \mathrm{C}$ NMR see Table 2; HRESIMS $\mathrm{m} / \mathrm{z} 459.3476$ (calcd for $\mathrm{C}_{29} \mathrm{H}_{47} \mathrm{O}_{4}, 459.3474$ ).

\subsubsection{Stoloniferone $\mathrm{K}(4)$}

Colorless amorphous solid; $[\alpha]_{\mathrm{D}}^{25}=+18.0\left(\mathrm{c}=0.1, \mathrm{CHCl}_{3}\right)$; UV $(\mathrm{MeOH}) \lambda_{\max } 223 \mathrm{~nm}(\log \varepsilon 3.38) ; \mathrm{IR} \nu_{\max } 3480,1657 \mathrm{~cm}^{-1} ;{ }^{1} \mathrm{H}$ NMR see Table $1 ;{ }^{13} \mathrm{C}$ NMR see Table 2; HRESIMS $\mathrm{m} / \mathrm{z} 469.3291$ (calcd for $\mathrm{C}_{28} \mathrm{H}_{46} \mathrm{O}_{4} \mathrm{Na}$, 469.3294).

\subsubsection{Stoloniferone L (5)}

Colorless amorphous solid; $[\alpha]_{\mathrm{D}}^{25}=-45.0\left(\mathrm{c}=0.1, \mathrm{CHCl}_{3}\right)$; IR $v_{\max } 3439,1703 \mathrm{~cm}^{-1} ;{ }^{1} \mathrm{H}$ NMR see Table $3 ;{ }^{13} \mathrm{C}$ NMR see Table 2; HRESIMS $\mathrm{m} / \mathrm{z} 445.3319$ (calcd for $\mathrm{C}_{28} \mathrm{H}_{45} \mathrm{O}_{4}, 445.3318$ ).

\subsubsection{Stoloniferone $\mathrm{M}(6)$}

Colorless amorphous solid; $[\alpha]_{\mathrm{D}}^{25}=-42.0\left(\mathrm{c}=0.1, \mathrm{CHCl}_{3}\right)$; IR $\nu_{\max } 3441,1705 \mathrm{~cm}^{-1} ;{ }^{1} \mathrm{H}$ NMR see Table $3 ;{ }^{13} \mathrm{C}$ NMR see Table 2; HRESIMS m/z 481.3292 (calcd for $\mathrm{C}_{29} \mathrm{H}_{46} \mathrm{O}_{4} \mathrm{Na}$, 481.3294).

\subsubsection{Stoloniferone $N(7)$}

Colorless amorphous solid; $[\alpha]_{D}^{25}=-23.0\left(c=0.1, \mathrm{CHCl}_{3}\right)$; IR $\nu_{\max } 3443,1705 \mathrm{~cm}^{-1} ;{ }^{1} \mathrm{H}$ NMR see Table $3 ;{ }^{13} \mathrm{C}$ NMR see Table 2; HRESIMS m/z 469.3292 (calcd for $\mathrm{C}_{28} \mathrm{H}_{46} \mathrm{O}_{4} \mathrm{Na}$, 469.3294 .

\subsubsection{Stoloniferone O (8)}

Colorless amorphous solid; $[\alpha]_{D}^{25}=+10.0\left(c=0.1, \mathrm{CHCl}_{3}\right)$; UV $(\mathrm{MeOH}) \lambda \max \mathrm{nm}(\log \varepsilon) 314$ (4.20); IR $v_{\max } 3409,1652 \mathrm{~cm}^{-1} ;{ }^{1} \mathrm{H}$ NMR see Table $4 ;{ }^{13} \mathrm{C}$ NMR see Table 2; HRESIMS m/z 449.3029 (calcd for $\mathrm{C}_{28} \mathrm{H}_{42} \mathrm{O}_{3} \mathrm{Na}$, 449.3031).

\subsubsection{Stoloniferone P (9)}

Colorless amorphous solid; $[\alpha]_{\mathrm{D}}^{25}=+9.2\left(c=0.1, \mathrm{CHCl}_{3}\right)$; UV (MeOH) $\lambda_{\max } \mathrm{nm}(\log \varepsilon) 313$ (4.12); IR $v_{\max } 3420,1656 \mathrm{~cm}^{-1} ;{ }^{1} \mathrm{H}$ NMR see Table $4 ;{ }^{13} \mathrm{C}$ NMR see Table 2; HRESIMS $\mathrm{m} / \mathrm{z} 449.3028$ (calcd for $\mathrm{C}_{28} \mathrm{H}_{42} \mathrm{O}_{3} \mathrm{Na}$, 449.3031).

\subsubsection{Stoloniferone $Q(10)$}

Colorless amorphous solid; $[\alpha]_{D}^{25}=+18.6\left(c=0.2, \mathrm{CHCl}_{3}\right)$; UV (MeOH) $\lambda_{\max } \mathrm{nm}(\log \varepsilon) 313$ (4.22); IR $v_{\max } 3380,1655 \mathrm{~cm}^{-1} ;{ }^{1} \mathrm{H}$ NMR see Table $4 ;{ }^{13} \mathrm{C}$ NMR see Table 2; HRESIMS m/z 463.3192 (calcd for $\mathrm{C}_{29} \mathrm{H}_{44} \mathrm{O}_{3} \mathrm{Na}$, 463.3188).

\subsection{Cytotoxicity testing}

P-388 cells were kindly supplied by J. M. Pezzuto, formerly of the Department of Medicinal Chemistry and Pharmacognosy, University of Illinois at Chicago; HT-29 cells were purchased from the American Type Culture Collection. Cytotoxic assays 
Table $1-{ }^{1} \mathrm{H}$ NMR spectroscopic data ${ }^{\mathrm{a}}(300 \mathrm{MHz})$ of $1-4$ in $\mathrm{CDCl}_{3}$

\begin{tabular}{|c|c|c|c|c|}
\hline & 1 & 2 & 3 & 4 \\
\hline 2 & $6.02 \mathrm{dd}(9.9,2.4)^{\mathrm{b}}$ & $6.01 \mathrm{dd}(9.9,2.4)$ & $6.02 \mathrm{dd}(9.9,2.4)$ & 6.02 dd $(9.9,2.4)$ \\
\hline 3 & $6.69 \mathrm{dt}(9.9,2.4)$ & $6.69 \mathrm{dt}(9.9,2.4)$ & $6.68 \mathrm{dt}(9.9,2.4)$ & $6.69 \mathrm{dt}(9.9,2.4)$ \\
\hline $4 \alpha$ & $2.22 \mathrm{~m}$ & $2.16 \mathrm{~m}$ & $2.08 \mathrm{~m}$ & $2.15 \mathrm{~m}$ \\
\hline $4 \beta$ & $3.43 \mathrm{dt}(19.8,2.4)$ & $3.43 \mathrm{dt}(19.8,2.4)$ & $3.42 \mathrm{dt}(19.8,2.4)$ & $3.44 \mathrm{dt}(19.8,2.4)$ \\
\hline 6 & $3.66 \mathrm{br} \mathrm{s}$ & $3.67 \mathrm{br} \mathrm{s}$ & 3.67 br s & $3.67 \mathrm{br} \mathrm{s}$ \\
\hline 7 & $1.82 \mathrm{~m}, 1.61 \mathrm{~m}$ & $1.70 \mathrm{~m}, 1.62 \mathrm{~m}$ & $1.82 \mathrm{~m}, 1.62 \mathrm{~m}$ & $1.70 \mathrm{~m}, 1.61 \mathrm{~m}$ \\
\hline 8 & $1.83 \mathrm{~m}$ & $1.95 \mathrm{~m}$ & $1.94 \mathrm{~m}$ & $1.91 \mathrm{~m}$ \\
\hline 9 & $2.00 \mathrm{~m}$ & $2.01 \mathrm{~m}$ & $2.02 \mathrm{~m}$ & $2.01 \mathrm{~m}$ \\
\hline 11 & $3.70 \mathrm{dt}(3.3,10.5)$ & $3.70 \mathrm{dt}(3.6,10.5)$ & $3.70 \mathrm{dt}(3.6,10.2)$ & $3.71 \mathrm{dt}(3.6,10.5)$ \\
\hline $12 \alpha$ & $1.43 \mathrm{~m}$ & $1.40 \mathrm{~m}$ & $1.44 \mathrm{~m}$ & $1.42 \mathrm{~m}$ \\
\hline $12 \beta$ & $2.34 \mathrm{dd}(12.6,3.3)$ & $2.36 \mathrm{dd}(12.3,3.6)$ & $2.36 \mathrm{dd}(12.3,3.6)$ & $2.37 \mathrm{dd}(12.6,3.6)$ \\
\hline 14 & $1.25 \mathrm{~m}$ & $1.23 \mathrm{~m}$ & $1.31 \mathrm{~m}$ & $1.25 \mathrm{~m}$ \\
\hline 15 & $1.52 \mathrm{~m}, 1.08 \mathrm{~m}$ & $1.62 \mathrm{~m}, 1.14 \mathrm{~m}$ & $1.63 \mathrm{~m}, 1.12 \mathrm{~m}$ & $1.62 \mathrm{~m}, 1.09 \mathrm{~m}$ \\
\hline 16 & $1.75 \mathrm{~m}, 1.27 \mathrm{~m}$ & $1.86 \mathrm{~m}, 1.30 \mathrm{~m}$ & $1.86 \mathrm{~m}, 1.42 \mathrm{~m}$ & $1.61 \mathrm{~m}, 1.35 \mathrm{~m}$ \\
\hline 17 & $1.28 \mathrm{~m}$ & $1.31 \mathrm{~m}$ & $1.38 \mathrm{~m}$ & $1.28 \mathrm{~m}$ \\
\hline 18 & $0.74 \mathrm{~s}$ & $0.78 \mathrm{~s}$ & $0.74 \mathrm{~s}$ & $0.77 \mathrm{~s}$ \\
\hline 19 & $1.48 \mathrm{~s}$ & $1.48 \mathrm{~s}$ & $1.48 \mathrm{~s}$ & $1.49 \mathrm{~s}$ \\
\hline 20 & $2.06 \mathrm{~m}$ & $1.43 \mathrm{~m}$ & $0.87 \mathrm{~m}$ & $1.38 \mathrm{~m}$ \\
\hline 21 & $1.05 \mathrm{~d}(6.6)$ & $0.99 \mathrm{~d}(6.3)$ & $0.97 d(6.6)$ & $0.96 \mathrm{~d}(6.0)$ \\
\hline 22 & $5.15 \mathrm{dd}(15.6,7.2)$ & $1.50 \mathrm{~m}, 1.18 \mathrm{~m}$ & $0.29 \mathrm{~m}$ & $1.42 \mathrm{~m}, 0.97 \mathrm{~m}$ \\
\hline 23 & $5.19 \mathrm{dd}(15.6,7.8)$ & $2.15 \mathrm{~m}, 1.93 \mathrm{~m}$ & $0.53 \mathrm{~m}$ & $1.41 \mathrm{~m}, 0.96 \mathrm{~m}$ \\
\hline 24 & $1.89 \mathrm{~m}$ & & $0.51 \mathrm{~m}$ & $1.24 \mathrm{~m}$ \\
\hline 25 & $1.50 \mathrm{~m}$ & $2.28 \mathrm{~m}$ & $1.70 \mathrm{~m}$ & $1.49 \mathrm{~m}$ \\
\hline 26 & $0.82 \mathrm{~d}(6.3)$ & $1.03 \mathrm{~d}(6.9)$ & $0.87 \mathrm{~d}(6.3)$ & $0.78 d(6.9)$ \\
\hline 27 & $0.84 \mathrm{~d}(6.3)$ & $1.03 \mathrm{~d}(6.9)$ & $0.88 d(6.0)$ & $0.86 d(6.9)$ \\
\hline 28 & $0.91 \mathrm{~d}(6.9)$ & $4.72 \mathrm{~s}, 4.66 \mathrm{~s}$ & $0.89 \mathrm{~d}(6.3)$ & $0.79 \mathrm{~d}(6.6)$ \\
\hline 29 & & & $0.13 \mathrm{~m}$ & \\
\hline $\mathrm{OH}-11$ & $5.19 \mathrm{~d}(5.7)$ & $5.03 \mathrm{~d}(6.3)$ & $4.99 \mathrm{~d}(5.7)$ & $4.99 \mathrm{~d}(5.7)$ \\
\hline
\end{tabular}

were carried out according to the previously described procedures [13].

\section{Results and discussion}

Compound 1 had a molecular formula of $\mathrm{C}_{28} \mathrm{H}_{44} \mathrm{O}_{4}$ as indicated by HRESIMS and ${ }^{13} \mathrm{C}$ NMR spectroscopic data. ${ }^{13} \mathrm{C}$ NMR and DEPT spectra of 1 exhibited the presence of six methyls, five $\mathrm{sp}^{3}$ methylenes, nine $\mathrm{sp}^{3}$ methines, four $\mathrm{sp}^{2}$ methines, three $\mathrm{sp}^{3}$ quaternary carbons and one $\mathrm{sp}^{2}$ quaternary carbon. The IR spectrum of 1 showed absorption due to a hydrogen bonded $\alpha, \beta$-unsaturated ketone $\left(1653 \mathrm{~cm}^{-1}\right)$. The presence of a conjugated enone system in 1 was also indicated by UV absorption at $224 \mathrm{~nm}(\log \varepsilon 3.56)$ as well as ${ }^{1} \mathrm{H}$ NMR [ $\delta 6.02(1 \mathrm{H}, \mathrm{dd}, J=9.9$, $2.4 \mathrm{~Hz}), 6.69(1 \mathrm{H}, \mathrm{dd}, J=9.9,2.4 \mathrm{~Hz})]$ (Table 1 ) and ${ }^{13} \mathrm{C}$ NMR $[\delta$ $128.9(\mathrm{CH}), 142.2$ (CH), 208.7 (qC)] spectra (Table 2). IR absorption at $3420 \mathrm{~cm}^{-1}$ and NMR signals at $\delta_{\mathrm{H}} 3.66(1 \mathrm{H}, \mathrm{br} \mathrm{s})$ and $3.70(1 \mathrm{H}, \mathrm{dt}, J=3.3,10.5 \mathrm{~Hz})$ as well as at $\delta_{\mathrm{C}} 74.7(\mathrm{CH})$ and 68.4 $(\mathrm{CH})$ indicated the presence of two secondary hydroxyl groups. The ${ }^{13} \mathrm{C}$ NMR data of 1 indicated some similarities to those of yonarasterol A [14], except for the absence of a secondary acetate at C-6. All C-H correlations of 1 were detected in the HSQC experiment. The ${ }^{1} \mathrm{H}-{ }^{1} \mathrm{H}$ COSY spectrum exhibited partial structures a, b, and c (Fig. 1). In the HMBC spectrum, partial structure a could be connected to $\mathrm{b}$ through three quaternary carbons (C-1, C-5 and C-10) and $\mathrm{H}_{3}-19$ (Fig. 1). Partial structure $\mathrm{b}$ could be connected to $\mathrm{c}$ through the remaining quaternary carbon (C-13) and $\mathrm{H}_{3}-18$. Based on these findings, the planar structure of 1 was concluded as in Fig. 1. The configuration at C-20 and C-24 was determined by comparison of ${ }^{13} \mathrm{C}$ NMR data with those of yonarasterol $\mathrm{A}$ and epimeric steroidal side chains $[14,15]$. The coupling constant between $\mathrm{H}-22$ and $\mathrm{H}-23$ $(J=15.6 \mathrm{~Hz})$ suggested the double bond to have $E$ configuration. The NOESY correlations (Fig. 2) observed between $\mathrm{H}-11$ and $\mathrm{H}$ $8, \mathrm{H}-11$ and $\mathrm{H}_{3}-18, \mathrm{H}-11$ and $\mathrm{H}_{3}-19, \mathrm{H}-3$ and $\mathrm{H}-2, \mathrm{H}-9$ and $\mathrm{H}-14$, $\mathrm{H}_{3}-18$ and $\mathrm{H}-8, \mathrm{H}_{3}-19$ and $\mathrm{H}-8, \mathrm{H}_{3}-18$ and $\mathrm{H}-20, \mathrm{H}-4 \beta$ and $\mathrm{H}_{3}-$ $19, \mathrm{H}-4 \alpha$ and $\mathrm{H}-6, \mathrm{H}_{3}-21$ and $\mathrm{H}-12 \beta, \mathrm{H}-9$ and $\mathrm{H}-12 \alpha$ indicated all trans configurations for ring fusions.

The spectroscopic data of 2-4 were analogous to those of 1, except for NMR signals due to the side chains. As shown

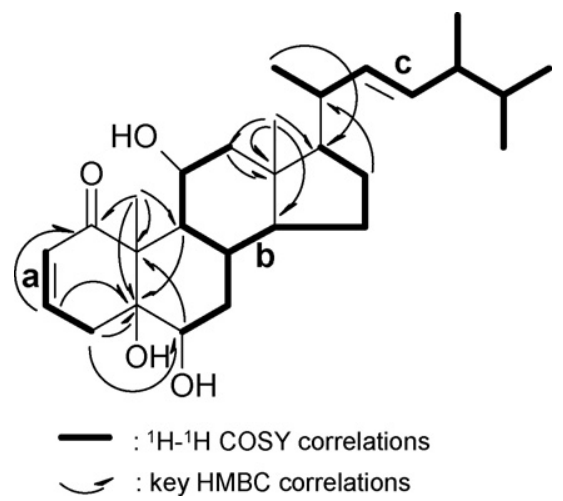

Fig. $1-{ }^{1} \mathrm{H}-{ }^{1} \mathrm{H}$ COSY and key HMBC correlations of 1 . 
Table $2-{ }^{13} \mathrm{C}$ NMR spectroscopic data ${ }^{\mathrm{a}}$ (75 MHz) of 1-10 in $\mathrm{CDCl}_{3}$

\begin{tabular}{|c|c|c|c|c|c|c|c|c|c|c|}
\hline & 1 & 2 & 3 & 4 & 5 & 6 & 7 & 8 & 9 & 10 \\
\hline 1 & 208.7 & 208.6 & 208.7 & 208.7 & 215.3 & 215.2 & 215.4 & 212.5 & 212.5 & 212.5 \\
\hline 2 & 128.9 & 128.9 & 128.9 & 128.9 & 47.1 & 47.1 & 47.1 & 126.9 & 126.9 & 126.9 \\
\hline 3 & 142.2 & 142.2 & 142.1 & 142.2 & 64.2 & 64.2 & 64.2 & 140.9 & 140.8 & 140.8 \\
\hline 4 & 36.8 & 36.8 & 36.3 & 36.8 & 40.9 & 40.9 & 40.9 & 119.0 & 119.0 & 119.0 \\
\hline 5 & 77.9 & 77.5 & 77.9 & 77.9 & 61.3 & 61.3 & 61.3 & 157.8 & 157.7 & 157.7 \\
\hline 6 & 74.7 & 74.7 & 74.7 & 74.7 & 60.9 & 61.0 & 61.0 & 73.7 & 73.7 & 73.7 \\
\hline 7 & 32.9 & 33.0 & 32.9 & 33.0 & 31.6 & 31.6 & 31.6 & 40.4 & 40.4 & 40.4 \\
\hline 8 & 28.5 & 28.4 & 28.4 & 28.4 & 28.0 & 28.5 & 28.0 & 29.5 & 29.5 & 29.5 \\
\hline 9 & 47.5 & 47.5 & 47.2 & 47.5 & 49.9 & 49.8 & 49.8 & 58.3 & 58.1 & 58.2 \\
\hline 10 & 54.0 & 54.0 & 54.3 & 54.0 & 51.8 & 51.7 & 51.7 & 55.4 & 55.4 & 55.4 \\
\hline 11 & 68.4 & 68.4 & 68.4 & 68.4 & 67.6 & 67.6 & 67.6 & 66.9 & 66.9 & 66.9 \\
\hline 12 & 51.0 & 51.2 & 51.2 & 51.2 & 49.2 & 49.2 & 49.3 & 49.4 & 49.6 & 49.5 \\
\hline 13 & 43.1 & 43.3 & 43.6 & 43.2 & 42.8 & 43.2 & 42.9 & 42.7 & 42.9 & 43.2 \\
\hline 14 & 56.0 & 55.9 & 55.6 & 55.9 & 55.5 & 55.2 & 55.4 & 55.0 & 54.9 & 54.6 \\
\hline 15 & 24.0 & 24.0 & 24.0 & 24.0 & 24.0 & 24.4 & 24.1 & 24.6 & 24.6 & 24.9 \\
\hline 16 & 28.8 & 28.5 & 28.5 & 28.5 & 28.6 & 29.8 & 28.2 & 28.7 & 28.3 & 28.6 \\
\hline 17 & 55.9 & 56.1 & 57.5 & 56.1 & 55.8 & 57.4 & 55.9 & 55.9 & 56.0 & 57.5 \\
\hline 18 & 13.8 & 13.6 & 13.1 & 13.6 & 13.0 & 12.6 & 12.8 & 13.2 & 13.1 & 12.9 \\
\hline 19 & 15.1 & 15.1 & 15.1 & 15.1 & 13.6 & 13.6 & 13.6 & 19.6 & 19.6 & 19.6 \\
\hline 20 & 40.3 & 35.9 & 39.8 & 36.3 & 40.1 & 40.0 & 36.1 & 40.2 & 35.8 & 40.1 \\
\hline 21 & 20.8 & 18.5 & 19.1 & 18.7 & 20.9 & 19.1 & 18.8 & 20.9 & 18.6 & 19.2 \\
\hline 22 & 135.6 & 34.6 & 25.2 & 33.6 & 135.4 & 25.2 & 33.6 & 135.5 & 34.6 & 25.2 \\
\hline 23 & 132.0 & 31.1 & 24.0 & 30.7 & 132.2 & 24.1 & 30.6 & 132.1 & 31.1 & 24.1 \\
\hline 24 & 42.8 & 156.9 & 45.0 & 39.1 & 42.8 & 45.0 & 39.1 & 42.8 & 156.8 & 45.0 \\
\hline 25 & 33.1 & 33.9 & 33.0 & 31.5 & 33.1 & 32.9 & 31.5 & 33.1 & 33.9 & 32.9 \\
\hline 26 & 19.7 & 21.9 & 18.6 & 17.7 & 19.7 & 18.6 & 17.7 & 19.7 & 21.9 & 18.6 \\
\hline 27 & 20.0 & 22.1 & 20.7 & 20.6 & 20.0 & 20.7 & 20.5 & 20.0 & 22.1 & 20.8 \\
\hline 28 & 17.7 & 106.0 & 15.9 & 15.5 & 17.6 & 15.8 & 15.5 & 17.7 & 106.1 & 15.9 \\
\hline 29 & & & 10.5 & & & 10.5 & & & & 10.5 \\
\hline
\end{tabular}

a Assigned by DEPT, COSY, HSQC, and HMBC experiments.

in Tables 1 and 2, signals due to an E disubstituted double bond were absent in 2 , but those due to an exo double bond [ $\delta_{\mathrm{H}} 4.72(1 \mathrm{H}, \mathrm{s})$ and $4.66(1 \mathrm{H}, \mathrm{s}) ; \delta_{\mathrm{C}} 156.9(\mathrm{qC})$ and $\left.106.0\left(\mathrm{CH}_{2}\right)\right]$ appeared in 2, similar to those observed for stoloniferone G.. ${ }^{11}$ Thus, the structure of 2 was concluded as a 22,23-dihydro24,28 -dehydro analog of 1 . NMR spectra of 3 suggested the $E$ disubstituted olefin in the side chain of 1 to be replaced by a 1,2-disubstituted cyclopropane $\left[\delta_{\mathrm{H}} 0.13(2 \mathrm{H}, \mathrm{m}), 0.29(1 \mathrm{H}, \mathrm{m})\right.$, and $0.53(1 \mathrm{H}, \mathrm{m}) ; \delta_{\mathrm{C}} 10.5\left(\mathrm{CH}_{2}\right), 25.2(\mathrm{CH})$ and $\left.24.0(\mathrm{CH})\right]$. HMBC analysis indicated that 3 has a cyclopropane at C-22 and C23. The configuration of four chiral centers (C-20, C-22, C-23, and $\mathrm{C}$-24) in the side chain was determined by comparison of ${ }^{13} \mathrm{C}$ NMR spectral data with those of stoloniferone- $d$, the stereostructure of which was established by X-ray crystallographic analysis [16]. NMR signals assigned to a $-\mathrm{CH}_{2} \mathrm{CH}_{2}-$ group at C22 and $\mathrm{C}-23\left[\delta_{\mathrm{C}} 33.6\left(\mathrm{CH}_{2}\right)\right.$ and $\left.30.7\left(\mathrm{CH}_{2}\right)\right]$ in 4 were observed instead of the $E$ disubstituted double bond in 1 as shown in

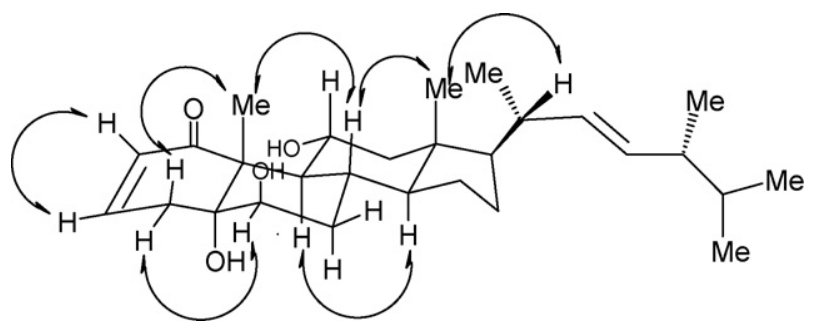

Fig. 2 - Selective NOESY correlations of 1.
Tables 1 and 2. Assignment of the configuration at C-20 and C24 of 4 was made by comparison of ${ }^{13} \mathrm{C}$ NMR data with those of yonarasterol B and epimeric steroidal side chains $[14,15]$.

Compound 5 was assigned a molecular formula of $\mathrm{C}_{28} \mathrm{H}_{44} \mathrm{O}_{4}$ as indicated by HRESIMS and ${ }^{13} \mathrm{C}$ NMR spectroscopic data. ${ }^{13} \mathrm{C}$ NMR and DEPT spectra of 5 showed the presence of six methyls, six $\mathrm{sp}^{3}$ methylenes, ten $\mathrm{sp}^{3}$ methines, two $\mathrm{sp}^{2}$ methines, three $\mathrm{sp}^{3}$ quaternary carbons and one $\mathrm{sp}^{2}$ quaternary carbon. The IR absorption at $1703 \mathrm{~cm}^{-1}$ and ${ }^{13} \mathrm{C} N M R$ signal (Table 2) at $\delta 215.3$ exhibited the presence of a ketone. IR absorption at $3439 \mathrm{~cm}^{-1}$ and NMR signals at $\delta_{\mathrm{H}} 4.19(1 \mathrm{H}, \mathrm{m})$ and $3.80(1 \mathrm{H}, \mathrm{dt}, J=4.5,10.5 \mathrm{~Hz})$ (Table 3 ) as well as at $\delta_{\mathrm{C}} 67.6$ $(\mathrm{CH})$ and $64.2(\mathrm{CH})$ indicated the presence of two secondary hydroxyl groups. The presence of a trisubstituted epoxy was shown by NMR signals at $\delta_{\mathrm{H}} 3.15\left(1 \mathrm{H}, \mathrm{br}\right.$ s) as well as at $\delta_{\mathrm{C}} 60.9$ $(\mathrm{CH})$ and $61.3(\mathrm{qC})$. The spectroscopic data of 5 were analogous to those of stoliferone $b$ [3], except for the data due to $\mathrm{C}-2$ and $\mathrm{C}-3$. The ${ }^{1} \mathrm{H}-{ }^{1} \mathrm{H}$ COSY spectrum showed partial structures $d, e$, and $\mathbf{f}$ (Fig. 3). In the HMBC spectrum, partial structure $d$ could be connected to e through three quaternary carbons (C-1, C-5 and $\mathrm{C}-10$ ) and $\mathrm{H}_{3}-19$ (Fig. 3). Partial structure e could be connected to $c$ through the remaining quaternary carbons (C-13) and $\mathrm{H}_{3}-18$. Based on this evidence, the planar structure of 5 was concluded as in Fig. 3. Configuration at C-20 and C-24 was determined by comparison of ${ }^{13} \mathrm{C}$ NMR data with those of 1 and epimeric steroidal side chains $[14,15]$. The coupling constant between $\mathrm{H}-22$ and $\mathrm{H}-23(\mathrm{~J}=15.6 \mathrm{~Hz})$ defined the double bond to have E configuration. The NOESY correlations (Fig. 4) 
Table $3-{ }^{1} \mathrm{H}$ NMR spectroscopic data ${ }^{\mathrm{a}}(300 \mathrm{MHz})$ of $5-7$ in $\mathrm{CDCl}_{3}$

\section{5}

6

7

\begin{tabular}{|c|c|c|c|}
\hline $2 \alpha$ & $3.05 \mathrm{dd}(13.5,6.9)^{\mathrm{b}}$ & $3.06 \mathrm{dd}(12.9,6.6)$ & $3.04 \mathrm{dd}(13.1,6.6)$ \\
\hline $2 \beta$ & $2.61 \mathrm{dd}(13.5,5.7)$ & $2.61 \mathrm{dd}(12.9,5.7)$ & $2.60 \mathrm{dd}(13.1,6.0)$ \\
\hline 3 & $4.19 \mathrm{~m}$ & $4.19 \mathrm{~m}$ & $4.18 \mathrm{~m}$ \\
\hline $4 \alpha$ & $1.68 \mathrm{~m}$ & $1.67 \mathrm{~m}$ & $1.68 \mathrm{~m}$ \\
\hline $4 \beta$ & $2.21 \mathrm{~m}$ & $2.20 \mathrm{~m}$ & $2.18 \mathrm{~m}$ \\
\hline 6 & $3.15 \mathrm{br} \mathrm{s}$ & $3.15 \mathrm{br} \mathrm{s}$ & $3.15 \mathrm{br} \mathrm{s}$ \\
\hline 7 & $2.20 \mathrm{~m}, 1.31 \mathrm{~m}$ & $2.20 \mathrm{~m}, 1.50 \mathrm{~m}$ & $2.21 \mathrm{~m}, 1.39 \mathrm{~m}$ \\
\hline 8 & $1.30 \mathrm{~m}$ & $1.28 \mathrm{~m}$ & $1.30 \mathrm{~m}$ \\
\hline 9 & $1.23 \mathrm{~m}$ & $1.39 \mathrm{~m}$ & $1.38 \mathrm{~m}$ \\
\hline 11 & $3.80 \mathrm{dt}(4.5,10.5)$ & $3.77 \mathrm{dt}(4.5,10.5)$ & $3.79 \mathrm{dt}(4.8,10.5)$ \\
\hline $12 \alpha$ & $1.20 \mathrm{~m}$ & $1.18 \mathrm{~m}$ & $1.19 \mathrm{~m}$ \\
\hline $12 \beta$ & $2.26 \mathrm{dd}(12.0,4.5)$ & $2.27 \mathrm{dd}(12.0,4.5)$ & $2.27 \mathrm{dd}(12.3,4.8)$ \\
\hline 14 & $1.39 \mathrm{~m}$ & $1.05 \mathrm{~m}$ & $1.07 \mathrm{~m}$ \\
\hline 15 & $1.58 \mathrm{~m}, 1.07 \mathrm{~m}$ & $1.62 \mathrm{~m}, 1.08 \mathrm{~m}$ & $1.63 \mathrm{~m}, 1.05 \mathrm{~m}$ \\
\hline 16 & $1.76 \mathrm{~m}, 1.28 \mathrm{~m}$ & $2.09 \mathrm{~m}, 1.46 \mathrm{~m}$ & $1.90 \mathrm{~m}, 1.30 \mathrm{~m}$ \\
\hline 17 & $1.09 \mathrm{~m}$ & $1.29 \mathrm{~m}$ & $1.20 \mathrm{~m}$ \\
\hline 18 & $0.67 \mathrm{~s}$ & $0.62 \mathrm{~s}$ & $0.66 \mathrm{~s}$ \\
\hline 19 & $1.45 \mathrm{~s}$ & $1.41 \mathrm{~s}$ & $1.41 \mathrm{~s}$ \\
\hline 20 & $2.01 \mathrm{~m}$ & $0.72 \mathrm{~m}$ & $1.34 \mathrm{~m}$ \\
\hline 21 & $1.03 \mathrm{~d}(6.6)$ & $0.93 d(6.9)$ & $0.92 \mathrm{~d}(6.3)$ \\
\hline 22 & $5.14 \mathrm{dd}(15.6,7.5)$ & $0.27 \mathrm{~m}$ & $1.38 \mathrm{~m}, 0.95 \mathrm{~m}$ \\
\hline 23 & $5.20 \mathrm{dd}(15.6,8.1)$ & $0.52 \mathrm{~m}$ & $1.30 \mathrm{~m}, 0.95 \mathrm{~m}$ \\
\hline 24 & $1.85 \mathrm{~m}$ & $0.50 \mathrm{~m}$ & $1.23 \mathrm{~m}$ \\
\hline 25 & $1.49 \mathrm{~m}$ & $1.68 \mathrm{~m}$ & $1.56 \mathrm{~m}$ \\
\hline 26 & $0.82 \mathrm{~d}(6.6)$ & $0.86 \mathrm{~d}(6.6)$ & $0.78 \mathrm{~d}(6.6)$ \\
\hline 27 & $0.82 \mathrm{~d}(6.6)$ & $0.86 \mathrm{~d}(6.6)$ & $0.85 d(6.6)$ \\
\hline 28 & $0.91 \mathrm{~d}(6.6)$ & $0.89 \mathrm{~d}(6.9)$ & $0.77 d(6.6)$ \\
\hline 29 & & $0.12 \mathrm{~m}$ & \\
\hline $\mathrm{OH}-11$ & $2.64 \mathrm{br} \mathrm{s}$ & $2.65 \mathrm{br} \mathrm{s}$ & $2.66 \mathrm{br} \mathrm{s}$ \\
\hline
\end{tabular}

a Assigned by COSY, HSQC, NOESY, and HMBC experiments. ${ }^{\mathrm{b}} \mathrm{J}$ values (in $\mathrm{Hz}$ ) in parentheses.

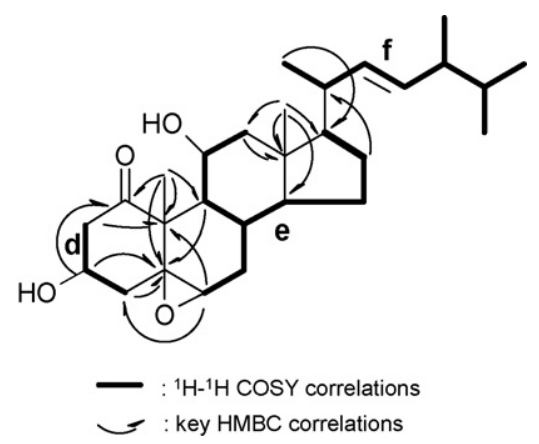

Fig. $3-{ }^{1} \mathrm{H}-{ }^{1} \mathrm{H}$ COSY and key HMBC correlations of 5.

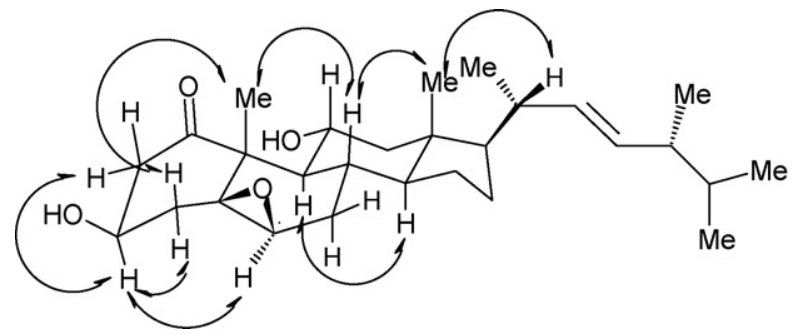

Fig. 4 - Selective NOESY correlations of 5.
Table $4-{ }^{1} \mathrm{H}$ NMR spectroscopic data ${ }^{\mathrm{a}}(300 \mathrm{MHz})$ of 8-10 in $\mathrm{CDCl}_{3}$

$$
8
$$

9

10

\begin{tabular}{|c|c|c|c|}
\hline 2 & $6.16 \mathrm{~d}(9.3)^{\mathrm{b}}$ & $6.16 \mathrm{~d}(9.3)$ & $6.17 \mathrm{~d}(9.3)$ \\
\hline 3 & $6.99 \mathrm{dd}(9.3,6.0)$ & $6.99 \mathrm{dd}(9.3,6.0)$ & $6.99 \mathrm{dd}(9.3,6.0)$ \\
\hline 4 & $6.18 \mathrm{~d}(6.0)$ & $6.19 \mathrm{~d}(6.0)$ & $6.19 \mathrm{~d}(6.0)$ \\
\hline 6 & $4.56 \mathrm{br} \mathrm{s}$ & $4.56 \mathrm{br} \mathrm{s}$ & $4.57 \mathrm{br} \mathrm{s}$ \\
\hline 7 & $2.08 \mathrm{~m}, 1.31 \mathrm{~m}$ & $2.03 \mathrm{~m}, 1.31 \mathrm{~m}$ & $2.03 \mathrm{~m}, 1.30 \mathrm{~m}$ \\
\hline 8 & $2.16 \mathrm{~m}$ & $2.15 \mathrm{~m}$ & $2.16 \mathrm{~m}$ \\
\hline 9 & $1.45 \mathrm{~m}$ & $1.43 \mathrm{~m}$ & $1.42 \mathrm{~m}$ \\
\hline 11 & $4.20 \mathrm{dt}(4.5,10.5)$ & $4.19 \mathrm{dt}(4.8,10.2)$ & $4.19 \mathrm{dt}(4.8,10.5)$ \\
\hline $12 \alpha$ & $1.26 \mathrm{~m}$ & $1.25 \mathrm{~m}$ & $1.23 \mathrm{~m}$ \\
\hline $12 \beta$ & $2.36 \mathrm{dd}(12.3,4.5)$ & $2.41 \mathrm{dd}(12.6,4.8)$ & $2.37 \mathrm{dd}(12.6,4.8)$ \\
\hline 14 & $1.15 \mathrm{~m}$ & $1.17 \mathrm{~m}$ & $1.13 \mathrm{~m}$ \\
\hline 15 & $1.58 \mathrm{~m}, 1.17 \mathrm{~m}$ & $1.62 \mathrm{~m}, 1.17 \mathrm{~m}$ & $1.62 \mathrm{~m}, 1.17 \mathrm{~m}$ \\
\hline 16 & $1.76 \mathrm{~m}, 1.21 \mathrm{~m}$ & $1.74 \mathrm{~m}, 1.34 \mathrm{~m}$ & $1.44 \mathrm{~m}$ \\
\hline 17 & $1.24 \mathrm{~m}$ & $1.23 \mathrm{~m}$ & $1.32 \mathrm{~m}$ \\
\hline 18 & $0.79 \mathrm{~s}$ & $0.79 \mathrm{~s}$ & $0.74 \mathrm{~s}$ \\
\hline 19 & $1.69 \mathrm{~s}$ & $1.70 \mathrm{~s}$ & $1.69 \mathrm{~s}$ \\
\hline 20 & $2.08 \mathrm{~m}$ & $1.45 \mathrm{~m}$ & $0.86 \mathrm{~m}$ \\
\hline 21 & $1.03 \mathrm{~d}(6.6)$ & $0.98 \mathrm{~d}(6.3)$ & $0.95 d(6.3)$ \\
\hline 22 & 5.15 dd $(15.6,7.2)$ & $1.55 \mathrm{~m}, 1.17 \mathrm{~m}$ & $0.28 \mathrm{~m}$ \\
\hline 23 & $5.21 \mathrm{dd}(15.6,7.8)$ & $2.09 \mathrm{~m}, 1.92 \mathrm{~m}$ & $0.52 \mathrm{~m}$ \\
\hline 24 & $1.89 \mathrm{~m}$ & $1.89 \mathrm{~m}$ & $0.51 \mathrm{~m}$ \\
\hline 25 & $1.49 \mathrm{~m}$ & $2.23 \mathrm{~m}$ & $1.68 \mathrm{~m}$ \\
\hline 26 & $0.83 d(6.6)$ & $1.02 \mathrm{~d}(6.3)$ & $0.86 d(6.6)$ \\
\hline 27 & $0.83 \mathrm{~d}(6.6)$ & $1.02 \mathrm{~d}(6.3)$ & $0.88 \mathrm{~d}(6.6)$ \\
\hline 28 & $0.90 \mathrm{~d}(6.9)$ & $4.71 \mathrm{~s}, 4.65 \mathrm{~s}$ & $0.91 \mathrm{~d}(6.3)$ \\
\hline 29 & & & $0.12 \mathrm{~m}$ \\
\hline $\mathrm{OH}-11$ & $4.12 \mathrm{br} \mathrm{s}$ & $4.15 \mathrm{br} \mathrm{s}$ & $4.15 \mathrm{br} \mathrm{s}$ \\
\hline
\end{tabular}

observed between $\mathrm{H}-11$ and $\mathrm{H}-8, \mathrm{H}-11$ and $\mathrm{H}_{3}-18, \mathrm{H}-11$ and $\mathrm{H}_{3}-$ $19, \mathrm{H}-3$ and $\mathrm{H}-6, \mathrm{H}-9$ and $\mathrm{H}-14, \mathrm{H}_{3}-18$ and $\mathrm{H}-8, \mathrm{H}_{3}-19$ and $\mathrm{H}-8$, $\mathrm{H}_{3}-18$ and $\mathrm{H}-20, \mathrm{H}-4 \beta$ and $\mathrm{H}_{3}-19, \mathrm{H}-4 \alpha$ and $\mathrm{H}-3, \mathrm{H}_{3}-21$ and $\mathrm{H}-$ $12 \beta, H-9$ and $H-12 \alpha$ proved the relative configuration for each ring junction and chiral center.

The spectroscopic data of 6 and 7 resembled those of 5 , except for NMR signals due to the side chains. NMR spectra of 6 indicated that the $E$ disubstituted olefin in the side chain of 5 was replaced by a 1,2-disubstituted cyclopropane $\left[\delta_{\mathrm{H}} 0.12(2 \mathrm{H}\right.$, $\mathrm{m}), 0.27(1 \mathrm{H}, \mathrm{m})$, and $0.52(1 \mathrm{H}, \mathrm{m}) ; \delta_{\mathrm{C}} 10.5\left(\mathrm{CH}_{2}\right), 25.2(\mathrm{CH})$ and $24.1(\mathrm{CH})]$. An HMBC experiment helped ascertain 6 to have a cyclopropane at C-22 and C-23. The stereochemistry of the four chiral centers (C-20, C-22, C-23, and C-24) in the side chain was confirmed by comparison of ${ }^{13} \mathrm{C}$ NMR data with those of 3. NMR signals assigned to a $-\mathrm{CH}_{2} \mathrm{CH}_{2}$ - group at C-22 and C-23 [ $\delta_{\mathrm{C}} 33.6\left(\mathrm{CH}_{2}\right)$ and $\left.30.6\left(\mathrm{CH}_{2}\right)\right]$ in 7 replaced the $E$ disubstituted double bond signals in 5 as shown in Tables 1 and 2 . The configuration at C-20 and C-24 of 7 was determined by comparison of ${ }^{13} \mathrm{C}$ NMR data with those of 4 and epimeric steroidal side chains [14,15].

Compound 8 gave a molecular formula of $\mathrm{C}_{28} \mathrm{H}_{42} \mathrm{O}_{3}$ as shown by HRESIMS and ${ }^{13} \mathrm{C}$ NMR spectroscopic data. The ${ }^{13} \mathrm{C}$ NMR and DEPT spectrum of 8 exhibited the presence of six methyls, four $\mathrm{sp}^{3}$ methylenes, nine $\mathrm{sp}^{3}$ methines, five $\mathrm{sp}^{2}$ methines, two $\mathrm{sp}^{3}$ quaternary carbons and two $\mathrm{sp}^{2}$ quaternary carbons. The IR spectrum of 8 showed absorption due to an $\alpha, \beta, \gamma, \delta$-unsaturated ketone $\left(1652 \mathrm{~cm}^{-1}\right)$. The presence of a conjugated enone system in 8 was also indicated by UV 


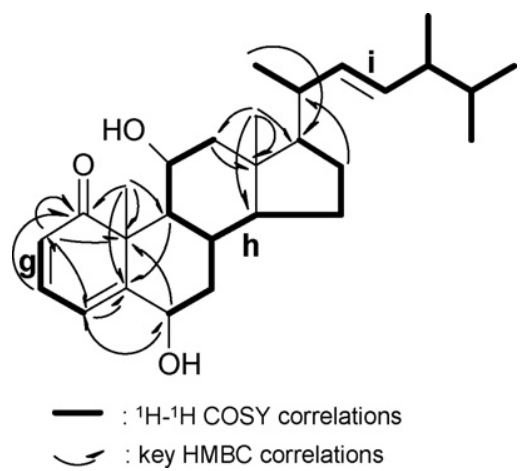

Fig. $5-{ }^{1} \mathrm{H}-{ }^{1} \mathrm{H}$ COSY and key HMBC correlations of 8.

absorptions at $314(\log \varepsilon 4.20) \mathrm{nm}$ as well as ${ }^{1} \mathrm{H}$ NMR $[\delta 6.16$ $(1 \mathrm{H}, \mathrm{d}, J=9.3 \mathrm{~Hz}), 6.18(1 \mathrm{H}, \mathrm{d}, J=6.0 \mathrm{~Hz}), 6.99(1 \mathrm{H}, \mathrm{dd}, J=9.3$, $6.0 \mathrm{~Hz}$ )] (Table 4) and ${ }^{13} \mathrm{C}$ NMR [ $\delta 119.0(\mathrm{CH}), 126.9(\mathrm{CH}), 140.9$ (CH), $157.8(\mathrm{C})$ ] spectra (Table 2). IR absorption at $3409 \mathrm{~cm}^{-1}$ and NMR signals at $\delta_{\mathrm{H}} 4.56(1 \mathrm{H}, \mathrm{brs})$ and $4.20(1 \mathrm{H}, \mathrm{dt}, J=10.5$, $4.5 \mathrm{~Hz})$ as well as at $\delta_{\mathrm{C}} 73.7(\mathrm{CH})$ and $66.9(\mathrm{CH})$ indicated the presence of two secondary hydroxyl groups. These data were analogous to those of stoloniferone E except for the side chain [11]. The ${ }^{1} \mathrm{H}-{ }^{1} \mathrm{H}$ COSY spectrum allowed construction of structures $\mathrm{g}, \mathrm{h}$, and $\mathrm{i}$ (Fig. 5). In the HMBC spectrum, partial structure $\mathrm{g}$ could be connected to $\mathrm{h}$ through three quaternary carbons (C-1, C-5 and C-10) and $\mathrm{H}_{3}-19$ (Fig. 5). Partial structure $\mathrm{h}$ could be connected to $\mathrm{i}$ through the remaining quaternary carbons (C-13) and $\mathrm{H}_{3}-18$. Based on these results, the planar structure of 8 was concluded as in Fig. 5. Configuration at C-20 and C-24 was determined by comparison of ${ }^{13} \mathrm{C}$ NMR data with those of 1 and epimeric steroidal side chains [14,15]. The coupling constant between $\mathrm{H}-22$ and $\mathrm{H}-23(\mathrm{~J}=15.6 \mathrm{~Hz})$ defined the double bond to have E configuration. The NOESY correlations observed between $\mathrm{H}-11$ and $\mathrm{H}-8, \mathrm{H}-11$ and $\mathrm{H}_{3}-18, \mathrm{H}-11$ and $\mathrm{H}_{3}-$ 19, $\mathrm{H}-4$ and $\mathrm{H}-6, \mathrm{H}-9$ and $\mathrm{H}-14, \mathrm{H}_{3}-18$ and $\mathrm{H}-8, \mathrm{H}_{3}-19$ and $\mathrm{H}-8$, $\mathrm{H}_{3}-18$ and $\mathrm{H}-20, \mathrm{H}_{3}-21$ and $\mathrm{H}-12 \beta, \mathrm{H}-9$ and $\mathrm{H}-12 \alpha$ indicated the relative configuration for each ring junction and chiral center.

The spectroscopic data of 9 and 10 were similar to those of 8, except for NMR signals due to the side chains. In the NMR data of 9 (Tables 2 and 4), signals assignable to an exo double bond $\left[\delta_{\mathrm{H}} 4.71(1 \mathrm{H}, \mathrm{s})\right.$ and $4.65(1 \mathrm{H}, \mathrm{s}) ; \delta_{\mathrm{C}} 156.8(\mathrm{qC})$ and 106.1 $\left(\mathrm{CH}_{2}\right)$ ] replaced the $\mathrm{E}$ disubstituted double bond in 8 . Therefore, the structure of 9 was concluded to be the 22,23-dihydro-24,28dehydro analog of 8 . NMR spectra of 10 indicated that the $E$ disubstituted double bond in the side chain of 8 was replaced by a 1,2-disubstituted cyclopropane $\left[\delta_{\mathrm{H}} 0.12(2 \mathrm{H}, \mathrm{m}), 0.28(1 \mathrm{H}\right.$, $\mathrm{m})$, and $0.52(1 \mathrm{H}, \mathrm{m}) ; \delta_{\mathrm{C}} 10.5\left(\mathrm{CH}_{2}\right), 25.2(\mathrm{CH})$ and $\left.24.1(\mathrm{CH})\right]$. HMBC analysis proved 10 to have a cyclopropane at C-22 and $\mathrm{C}-23$. Assignment of the configuration of four chiral centers (C-20, C-22, C-23, and C-24) in the side chain was made by comparison of ${ }^{13} \mathrm{C}$ NMR data with those of 3 .

The cytotoxicity of compounds $1-10$ is shown in Table 5. Compounds 1-3, 5, 6, and 8-10 exhibited cytotoxicity against HT-29 cell line. Compounds 1-10 exhibited cytotoxicity against P-388 cell line. All compounds showed stronger cytotoxicity than compounds 1-7 may be due to the presence of $\alpha, \beta, \gamma$, $\delta$-unsaturated carbonyl moiety in the molecule. Compounds
Table 5 - Cytotoxicity ${ }^{\mathrm{a}}$ of 1-10

\begin{tabular}{lcc}
\hline \multirow{2}{*}{ Compounds } & \multicolumn{2}{c}{ Cell lines IC $50(\mu \mathrm{g} / \mathrm{ml})$} \\
\cline { 2 - 3 } & HT-29 & P-388 \\
\hline 1 & 1.5 & 1.2 \\
2 & 1.8 & 1.5 \\
3 & 2.6 & 2.2 \\
4 & 4.8 & 3.6 \\
5 & 2.9 & 1.8 \\
6 & 3.2 & 2.4 \\
7 & 6.8 & 3.9 \\
8 & 0.2 & 0.1 \\
9 & 0.3 & 0.2 \\
10 & 0.5 & 0.3 \\
\hline \multirow{2}{*}{ a For significant activity of pure compounds, an $\mathrm{IC}_{50}$ of $\leq 4.0 \mu \mathrm{g} / \mathrm{ml}$} \\
\multicolumn{2}{c}{} \\
is required.
\end{tabular}

4 and 7 possessing cholestane side chain were less cytotoxic to HT-29 cell line.

\section{Acknowledgments}

Financial support was provided by Ministry of Education (96C030313) and National Science Council of Taiwan (NSC 952323-B-110-003) awarded to C.Y. Duh.

\section{REFERENCES}

[1] Honda A, Mori Y, Iguchi K, Yamada Y. Structure requirements for antiproliferative and cytotoxic activities of marine coral prostanoids from the Japanese stolonifer Clavularia viridis against human myeloid leukemia cells in culture. Prostaglandins 1988;36:621-30.

[2] Bader T, Yamada Y, Ankel H. Antiviral activity of the prostanoid clavulone II against vesicular stomatitis virus. Antiviral Res 1991;16:341-55.

[3] Kobayashi M, Lee NK, Son BW, Kyogoku Y, Yoshihara K, Kitagawa I. Stoloniferone -A, -B, -C, and -D, four cytotoxic steroids from the Okinawan soft coral Clavularia viridis. Tetrahedron Lett 1984;51:5925-8.

[4] Iwashima M, Okamoto K, Iguchi K. Clavirins, a new type of marine oxylipins with growth-inhibitory activity from the Okinawan soft coral, Clavularia viridis. Tetrahedron Lett 1999;40:6455-9.

[5] Iguchi K, Kaneta S, Mori K, Yamada Y, Honda A, Mori Y. Chlorovulones, new halogenated marine prostanoids with an antitumor activity from the stolonifer Clavularia viridis Quoy and Gaimard. Tetrahedron Lett 1985;26:5787-90.

[6] Watanabe K, Sekine M, Takahashi H, Iguchi K. New halogenated marine prostanoids with cytotoxic activity from the Okinawan soft coral Clavularia viridis. J Nat Prod 2001;64:1421-5.

[7] Iguchi K, Kaneta S, Mori K, Yamada Y, Honda A, Mori Y, Bromovulone I, iodovulone I. Unprecedented brominated and iodinated marine prostanoids with antitumor activity isolated from the Japanese stolonifer Clavularia viridis Quoy and Gaimard. J Chem Soc Chem Commun 1986;12:981-2.

[8] Shen YC, Cheng YB, Lin YC, Guh JH, Teng CM, Ko CL. New prostanoids with cytotoxic activity from Taiwanese octocoral Clauularia viridis. J Nat Prod 2004;67:542546.

[9] Iguchi K, Kaneta S, Mori K, Yamada Y. A new marine epoxy prostanoid with an antiproliferative activity from the 
stolonifer Clavularia viridis Quoy and Gaimard. Chem Pharm Bull 1987;35:4375-6.

[10] Watanabe K, Iwashima M, Iguchi K. New bioactive marine steroids from the Okinawan soft coral Clavularia viridis. Steroids 1996;61:439-46.

[11] Duh CY, El-Gamal AAH, Chu CJ, Wang SK, Chen HJ, Dai CF. New cytotoxic constituents from the Formosan soft corals Clauularia viridis and Clavularia violacea. J Nat Prod 2002;65:1535-9.

[12] Geran RI, Greenberg NH, MacDonald MM, Schumacher AM, Abbott BJ. Protocols for screening chemical agents and natural products against animal tumors and other biological systems. Cancer Chemother Rep 1972; 3:1-91.

[13] Hou RS, Duh CY, Chiang MY, Lin CN. Sinugibberol, a new cytotoxic cembranoid diterpene from the soft coral Sinularia gibberosa. J Nat Prod 1995;58:1126-30.

[14] Iwashima M, Nara K, Iguchi K. New marine steroids, yonarasterols, isolated from the Okinawan soft coral, Clavularia viridis. Steroids 2000;65:130-7.

[15] Wright JLC, McInnes AG, Shimizu S, Smith DG, Walter JA, Idler D, Khalil W. Identification of C-24 alkyl epimers of marine sterols by ${ }^{13} \mathrm{C}$ nuclear magnetic resonance spectroscopy. Can J Chem 1978;56:1898-905. 